\title{
Insight into excitation energy and structure effects in fission from isotopic information in fission yields
}

\author{
D. Ramos,,${ }^{1, *}$ M. Caamaño, ${ }^{1}$ F. Farget,${ }^{2, \dagger}$ C. Rodríguez-Tajes, ${ }^{2}$ L. Audouin, ${ }^{3}$ J. Benlliure, ${ }^{1}$ E. Casarejos, ${ }^{4}$ E. Clement,${ }^{2}$ \\ D. Cortina, ${ }^{1}$ O. Delaune, ${ }^{2,}$ X. Derkx,${ }^{5}$ A. Dijon, ${ }^{2}$ D. Doré,${ }^{6}$ B. Fernández-Domínguez, ${ }^{1}$ G. de France, ${ }^{2}$ A. Heinz,${ }^{7}$ B. Jacquot, ${ }^{2}$ \\ C. Paradela, ${ }^{1,}$ M. Rejmund ${ }^{2}$ T. Roger, ${ }^{2}$ M.-D. Salsac, ${ }^{6}$ and C. Schmitt ${ }^{2, \|}$ \\ ${ }^{1}$ Universidade de Santiago de Compostela, E-15706 Santiago de Compostela, Spain \\ ${ }^{2}$ GANIL, CEA/DSM-CNRS/IN2P3, BP 55027, F-14076 Caen Cedex 5, France \\ ${ }^{3}$ IPN Orsay, Université de Paris Sud-CNRS/IN2P3, F-91406 Orsay Cedex, France \\ ${ }^{4}$ Universidade de Vigo, E-36310 Vigo, Spain \\ ${ }^{5}$ LPC Caen, Université de Caen Basse-Normandie-ENSICAEN-CNRS/IN2P3, F-14050 Caen Cedex, France \\ ${ }^{6}$ CEA Saclay, DMS/IRFU/SPhN, 91191 Gif-sur-Yvette Cedex, France \\ ${ }^{7}$ Chalmers University of Technology, SE-41296 Göteborg, Sweden
}

(Received 23 November 2018; published 14 February 2019)

\begin{abstract}
The use of the inverse kinematics technique and a magnetic spectrometer permits the simultaneous measurement of proton and neutron content of full fission fragment distributions. This paper reports new measurements of five fissioning systems - ${ }^{238} \mathrm{U},{ }^{239} \mathrm{~Np},{ }^{240} \mathrm{Pu},{ }^{244} \mathrm{Cm}$, and ${ }^{250} \mathrm{Cf}$ - produced in inelastic scattering, transfer, and fusion reactions at different excitation energies. As a result, isotopic, elemental, and isotonic fission yields are presented. The contribution of the different fission modes and the proton even-odd effect are studied. Structure effects are investigated by means of the neutron excess and the total neutron multiplicity as a function of the fragment atomic number.
\end{abstract}

DOI: 10.1103/PhysRevC.99.024615

\section{INTRODUCTION}

Nuclear fission is a complex process that involves a collective motion of nucleons inside the nucleus and, at the same time, the single-particle shell structure of the nucleons strongly influences the path followed by the system down to scission [1-3]. Fission-fragment distributions reflect both macroscopic and microscopic aspects of the process and, hence, they provide key observables in the modeling of fission.

The potential energy surface affecting the deforming nucleus cannot be directly observed. Instead, models describe this surface based on observables from both incoming and outgoing channels [4-6]. The controversy between independent measurements of fragment mass [7] and recent nuclearcharge distributions [8] shows how difficult it is to describe the evolution along this potential energy.

\footnotetext{
"Present address: IPN Orsay, Université de Paris Sud-CNRS/ IN2P3, F-91406 Orsay Cedex, France; ramosdoval@ipno.in2p3.fr

†Present address: LPC Caen, Université de Caen BasseNormandie-ENSICAEN-CNRS/IN2P3, F-14050 Caen Cedex, France.

${ }^{\ddagger}$ Present address: CEA/DAM/DIF, F-91297 Arpajon, France.

${ }^{\S}$ Present address: EC-JRC, Institute for Reference Materials and Measurements, Retieseweg 1111, B-2440 Geel, Belgium.

"Present address: IPHC Strasbourg, Université de StrasbourgCNRS/IN2P3, F-67037 Strasbourg Cedex 2, France.
}

Final fission fragments are also affected by dynamical effects on the process, such as dissipation $[9,10]$ which is related to the viscosity of cold nuclear matter. This limits the accuracy of models based only on statistical scission-point configurations. In particular, the even-odd staggering observed in fission-fragment distributions appears to be related to these dynamical effects $[11,12]$ : the intrinsic excitation energy gained along the way to scission, together with the influence of the level density of each prefragment, is reflected in the rupture of pairs of nucleons along the way to the scission point.

In the fission process, the system reaches the scission point with a certain amount of excitation energy that is released by the fragments by neutron evaporation and $\gamma$ emission. The sharing of this total excitation energy between both fragments results in neutron- and $\gamma$-multiplicity distributions that cannot be modeled with the traditional understanding of the energy partition based on Fermi-gas level densities at constant temperature [13-16]. Instead, models such as FIFRELIN [17] need to introduce a mass-dependent temperature partition in order to reproduce the neutron-multiplicity distributions. In addition, an unbalanced-temperature process was recently suggested in which a continuous flux of energy would lead to a cold light fragment and a hot heavy partner [18].

Historically, the accessible experimental techniques limited the number of observables and systems to investigate, making it difficult to isolate the contribution of specific nuclear properties. For instance, fragment mass distributions were widely studied for decades, however, both proton and 
neutron contents cannot be disentangled from these measurements. The inverse kinematics technique opens the possibility to measure fragment nuclear-charge distributions [19] with full coverage and much higher accuracy than previous attempts in direct kinematics [20-24] and through $\gamma$ spectroscopy $[25,26]$.

Using the combination of the inverse kinematics and magnetic spectrometers provides access to full isotopic fragment distributions and to unstable fissioning systems [27-29] with new information, such as correlations between different observables, that permits to constrain current models.

Transfer- and fusion-induced fission proved to be suitable surrogate mechanisms to populate radioactive nuclei with control of their excitation energy. They are extensively used nowadays to measure different observables such as fission probabilities and fragment mass distributions [30-36].

This paper presents a study of the fission process based on transfer- and fusion-induced fission reactions within the fission campaign at GANIL using the VAMOS spectrometer. Details about the experimental setup and analysis can be found in Ref. [37]. With this technique, the simultaneous measurement of proton and neutron content of post-neutron evaporation fission fragments is achieved. New observables are addressed, such as isotonic distributions and the correlation between protons and neutrons within the fragments.
Different aspects of the process are investigated. In Sec. II, isotopic fission yields and the impact of the excitation energy are presented and compared with previous measurements. In Sec. III, elemental fission yields allow the study of the contribution of the different fission modes, where the proton even-odd staggering provides information about the interplay between the dynamics of the process and the level density of the nascent fragments. In Secs. IV-VI, the connection between collective motion and structure effects is investigated in terms of isotonic distributions, neutron excess of fission fragments, and neutron multiplicity, respectively. In Sec. VII, the conclusions of this work are reported.

\section{ISOTOPIC FISSION-FRAGMENT YIELDS}

Full isotopic distributions of fission fragments have been recently measured [37] in an experiment where the fissioning systems- ${ }^{238} \mathrm{U},{ }^{239} \mathrm{~Np},{ }^{240} \mathrm{Pu},{ }^{244} \mathrm{Cm}$, and ${ }^{250} \mathrm{Cf}$-were produced through inelastic scattering, multinucleon transfer, and fusion reactions using a beam of ${ }^{238} \mathrm{U}$ at $6.14 A \mathrm{MeV}$ and a ${ }^{12} \mathrm{C}$ target. The excitation energy $\left(E_{x}\right)$ distributions, measured in inelastic and transfer reactions, were centered at $\left\langle E_{x}\right\rangle=$ 7.4 MeV, 7.5 MeV, 10.7 MeV, and 23.0 MeV, respectively, with a full width at half-maximum of approximately $8 \mathrm{MeV}$ [31]. Concerning ${ }^{250} \mathrm{Cf}$, the fusion reaction fixes its mean

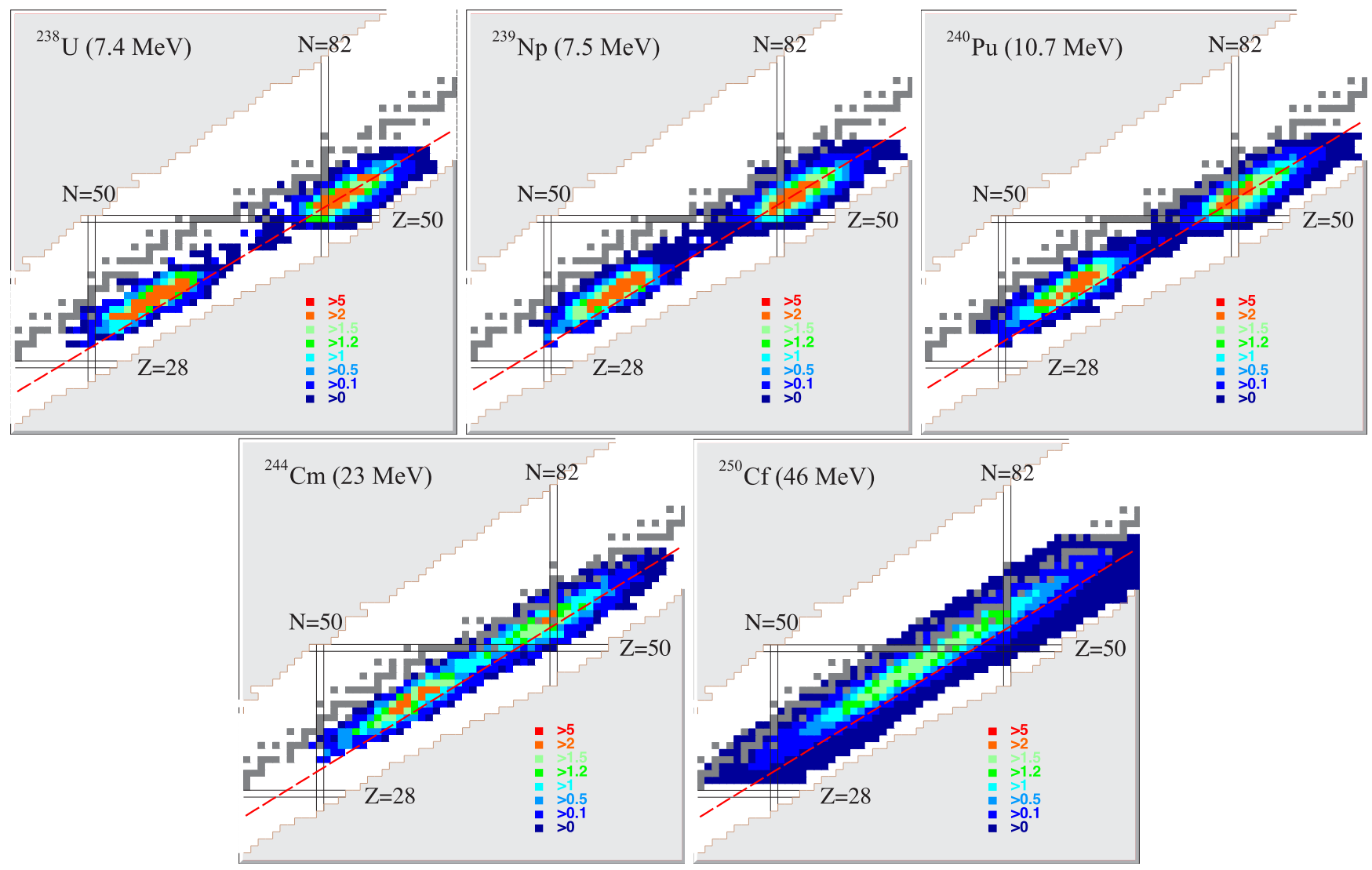

FIG. 1. Measured isotopic fission-fragment yields as a function of proton and neutron numbers. The valley of stability, shown as grey squares, and both the $n$-rich and the $n$-deficient limits of known nuclei, as well as the spherical closed shells $Z=28,50$, and $N=50,82$, are displayed. Dashed lines represent the $N / Z$ of the fissioning systems. The average initial excitation energy of each fissioning system is indicated in parentheses. 
energy $\left\langle E_{x}\right\rangle=46.0 \mathrm{MeV}$, with a full width at half-maximum of $0.6 \mathrm{MeV}$ due to the target thickness of $100 \mu \mathrm{g} / \mathrm{cm}^{2}$.

Figure 1 presents the isotopic fission yields of the five systems in terms of proton versus neutron numbers. The spherical closed shells $Z=28,50$, and $N=50,82$, are displayed, as well the $n$-rich and $n$-deficient limits of known nuclei on each side of the valley of stability. The colors represent the production of each isotope in units of normalized yields $\left[\sum_{Z, N} Y(Z, N)=200\right]$. These figures show that mostly neutron-rich isotopes with respect to the stability are produced, proving that fission is an efficient process to produce very $n$-rich nuclei. Two well-separated groups are observed in the systems at low excitation energy, with a deep valley for fission fragments with about equal masses that is absent in systems at higher excitation energy.

The red dashed lines represent the neutron excess $(N / Z)$ of the corresponding compound nuclei. Systems at low excitation energy show heavy fragments with a similar neutron excess to that of the fissioning system and less $n$-rich fragments in the light group. This difference between heavy and light fragments disappears with increasing $E_{x}$ and both are less $n$-rich than the fissioning system with a rather constant shift. The neutron excess is discussed in detail in Sec. V.

Figure 2 presents the isotopic distributions corresponding to symmetric splits. The isotopic fission yields of $Z=48,49$, and 50 are displayed. For $Z=48$ and 49 , only the systems with sufficient $E_{x}$ show considerable production. Whereas for $\mathrm{Sn}$ isotopes, associated with the proton magic number $Z=50,{ }^{238} \mathrm{U},{ }^{239} \mathrm{~Np}$, and ${ }^{240} \mathrm{Pu}$ show larger production with respect to ${ }^{250} \mathrm{Cf}$ and ${ }^{244} \mathrm{Cm}$, than for the previous elements, with a clear shift towards $n$-rich isotopes.

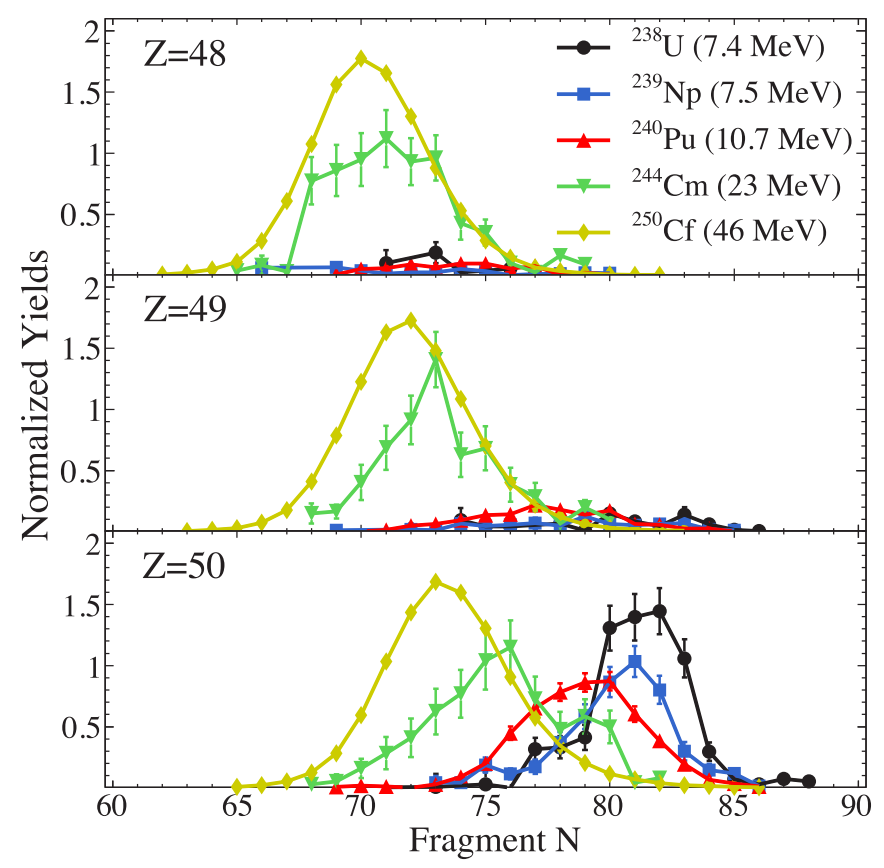

FIG. 2. Measured isotopic fission-fragment distributions of the elements $Z=48,49$, and 50, produced in the different fissioning systems. The corresponding average excitation energies are indicated in parentheses.

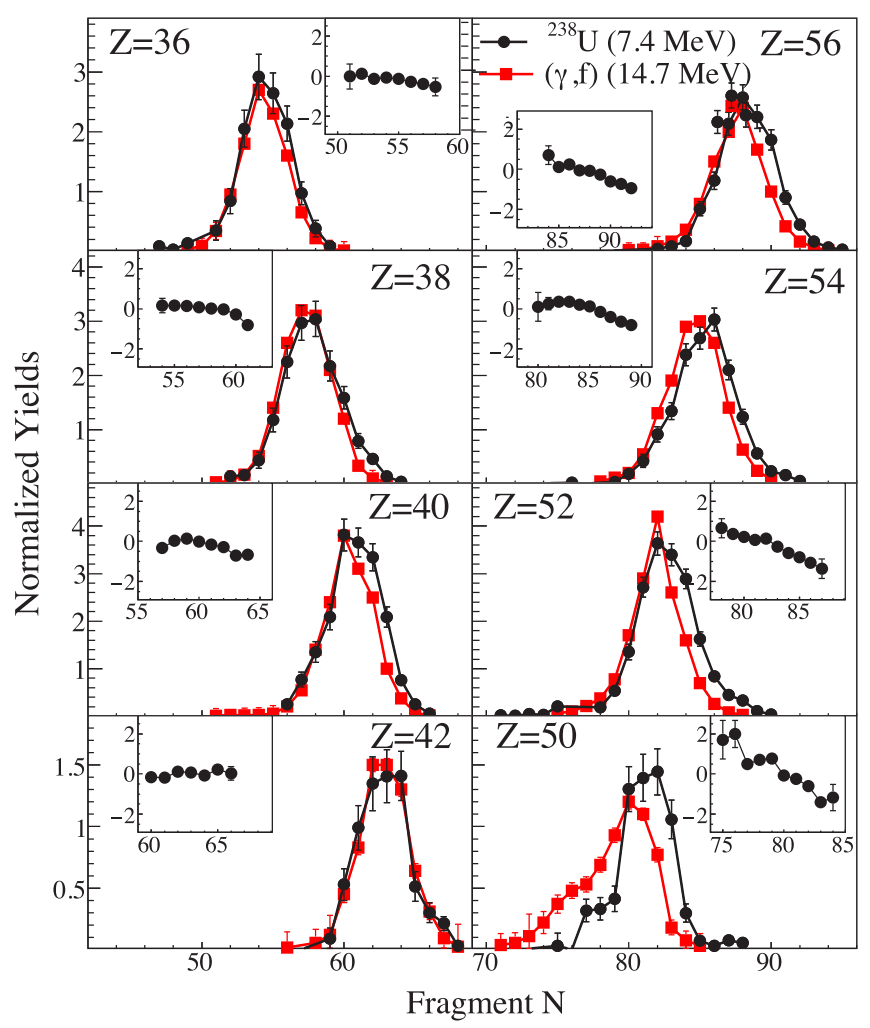

FIG. 3. Isotopic fission-fragment distributions of fissionfragment pairs with $Z=(36,56),(38,54),(40,52)$, and $(42,50)$ of ${ }^{238} \mathrm{U}$. Present data (in black) is compared with previous measurements employing Coulomb-induced fission (in red) from Ref. [29]. Insets: relative yield difference between both sets of data, $2\left(Y^{r}-Y^{b}\right) /\left(Y^{r}+Y^{b}\right)$.

The position of the yields around $N \approx 82$ for ${ }^{238} \mathrm{U}$ and ${ }^{239} \mathrm{~Np}$ reflects that the $Z=50$ split at scission is driven by the doubly magic nucleus ${ }^{132} \mathrm{Sn}$ at low $E_{x}$, followed by low neutron evaporation. $\operatorname{In}{ }^{250} \mathrm{Cf}$, the maximum around $N \approx 73$ is not caused by the influence of ${ }^{132} \mathrm{Sn}$, considering the number of evaporated neutrons for $Z=50$ is $v \approx 4$ [38], instead, the additional $E_{x}$ feeds symmetric splits and increases the contribution of the symmetric component. The asymmetry of the distributions also shows an evolution from ${ }^{238} \mathrm{U}$, with a tail at lower $N$, to ${ }^{250} \mathrm{Cf}$, with a tail at higher $N$. These asymmetric shapes may be explained as the coexistence of both, symmetric and asymmetric fission, centered at different $N$. This is consistent with the evolution of the other measured systems, where distributions mark the smooth transition from more to less neutron-rich maxima for increasing $E_{x}$. The coexistence is particularity clear in ${ }^{244} \mathrm{Cm}$, where both symmetric and asymmetric components show strong overlapping contributions due to its intermediate $E_{x}$ and its intermediate neutron richness. This is consistent with the fission modes, presented in the next section.

The impact of $E_{x}$ on fission of ${ }^{238} \mathrm{U}$ is presented in Fig. 3, where present data are compared with data from Coulombinduced fission [29], at $7 \mathrm{MeV}$ higher $E_{x}$. The most produced $Z$ pairs are shown in parallel, with insets showing the relative production between both sets of data, $2\left(Y^{r}-Y^{b}\right) /\left(Y^{r}+Y^{b}\right)$, 
where $Y^{r}$ are the yields of Coulomb-induced fission and $Y^{b}$ are the present yields.

Both sets agree well for the light fragments while, for the heavy fragments, there is a clear shift of the distributions to less neutron-rich isotopes for increasing $E_{x}$. This is reflected in the insets with a rather small slope for light fragments and a pronounced negative slope for heavy fragments. This observation indicates that the excess of $E_{x}$ is mainly exciting the heavy fragment, then released through neutron evaporation. This is consistent with previous measurements $[14,15]$ and with an unbalanced-temperature energy-sharing process [18].

The fact that no significant variations are observed in the light fragments indicates that the scission-point configuration for asymmetric splits remains almost unaffected by this excess of $E_{x}$. An interesting case is the pair $Z=(42,50) . Z=50$ is expected to have a low deformation at scission [39], understood as an effect of spherical shells, and any additional $E_{x}$ would modify such a configuration. However, the similar isotopic distributions of $Z=42$ for both $E_{x}$ suggest that the scission configuration is similar, and that the excess of energy is taken by the heavy fragment, despite its magicity. The large difference in $Z=50$ is produced, therefore, by the additional post-scission neutron evaporation. This shows a certain robustness of shell effects in the potential energy surface with increasing excitation energy.

\section{NUCLEAR CHARGE DISTRIBUTIONS: FISSION MODES AND EVEN-ODD STAGGERING}

Proton evaporation is strongly inhibited in this experiment due to the limited excitation energy, high proton-separation energies, and Coulomb barriers, hence elemental yields reflect the scission configuration. In this section, the proton content of the fission fragments is investigated. In Sec. III A, the fission-fragment production is described as a function of fission modes, and in Sec. IIIB, the dynamics of the fission process is investigated through the proton even-odd staggering.

\section{A. Fission modes}

Following the description of Brosa et al. [3], fission modes are the consequence of different paths along the potential energy surface as a function of the collective degrees of freedom. In this framework, the symmetric split is described by one symmetric fission mode, the Super Long (SL) mode, while asymmetric fission can be described mainly with two fission modes: the Standard I (SI) mode represents splits where the heavy fragment is rather spherical, driven by the doubly magic nucleus ${ }^{132} \mathrm{Sn}$ in actinides; the Standard II (SII) mode describes more asymmetric splits with well-deformed heavy fragments, centered around $Z \approx 55$.

The measured elemental-yield distributions were fitted to three Gaussian functions accounting for each fission mode, as shown in Fig. 4. An additional Superasymmetric mode (SA), also described in Ref. [3], was introduced in order to reproduce the very asymmetric splits of ${ }^{240} \mathrm{Pu}$ and ${ }^{244} \mathrm{Cm}$, with a very low contribution in both cases. The fits were constrained by two conditions: the amplitude of the SL mode

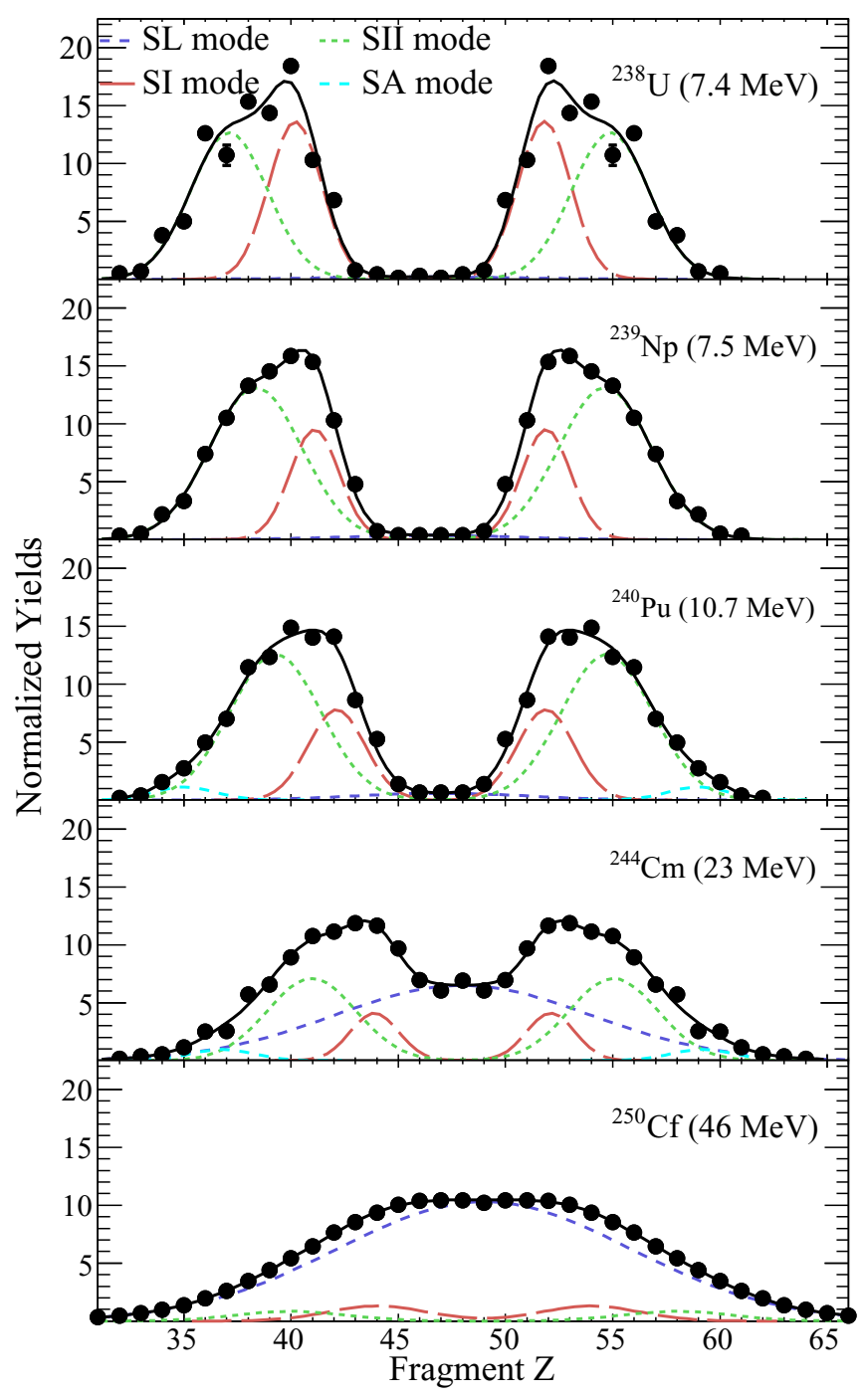

FIG. 4. Elemental fission-fragment yields fitted to the fission modes. Super Long (SL), Standard I (SI), Standard II (SII), and Superasymmetric (SA) fission modes are presented. See the text for details.

was fixed to the height of the distribution at symmetry and its standard deviation was fixed to 4 units in the systems at low $E_{x}$ $(\mathrm{U}, \mathrm{Np}$, and $\mathrm{Pu}$ ), following a general trend observed in lighter systems [19].

Table I summarizes the resulting parameters of the fission modes. The positions of the SI and SII modes remain rather constant at $Z_{S I} \approx 52$ and $Z_{S I I} \approx 55$, consistent with previous measurements of lighter systems [8], while in ${ }^{250} \mathrm{Cf}$ slightly higher values were obtained, $Z_{S I} \approx 54$ and $Z_{S I I} \approx 58$, probably affected by the large contribution of the SL mode. The larger width of SII with respect to SI is consistent with a less compact configuration at scission, where the stochastic process of neck rupture [3] favors a broadening of the fissionmode distribution.

In Fig. 5, the yields of the fission modes are presented as function of $E_{x}$ (top) and as a function of the fissility parameter $\left(Z^{2} / A\right)$ of each fissioning system (bottom). There is a strong correlation between the increasing $E_{x}$ and the increasing SL 
TABLE I. Information on the elemental fission modes. Positions, widths (standard deviations), and yields are presented for each fissioning system. The average excitation energies and their standard deviations are shown in parentheses.

\begin{tabular}{lcccc}
\hline \hline \multicolumn{5}{c}{ Fission modes parameters } \\
\hline System & Mode & Position & Width $(\sigma)$ & Yield (\%) \\
\hline${ }^{238} \mathrm{U}(7.4 \pm 3.0)$ & SI & $51.8 \pm 0.1$ & $1.23 \pm 0.04$ & $42.0 \pm 3.1$ \\
& SII & $54.9 \pm 0.2$ & $1.79 \pm 0.08$ & $56.8 \pm 3.4$ \\
& SL & 46 & 4 & $1.09 \pm 0.41$ \\
${ }^{239} \mathrm{~Np}(7.5 \pm 2.8)$ & SI & $51.9 \pm 0.1$ & $1.15 \pm 0.05$ & $27.4 \pm 2.1$ \\
& SII & $54.6 \pm 0.1$ & $2.15 \pm 0.04$ & $70.7 \pm 2.4$ \\
& SL & 46.5 & 4 & $1.83 \pm 0.11$ \\
${ }^{240} \mathrm{Pu}(10.7 \pm 3.0)$ & SI & $51.9 \pm 0.1$ & $1.33 \pm 0.05$ & $26.1 \pm 2.9$ \\
& SII & $54.7 \pm 0.1$ & $2.12 \pm 0.07$ & $67.0 \pm 3.0$ \\
& SA & $59.0 \pm 0.1$ & $1.44 \pm 0.07$ & $4.08 \pm 0.52$ \\
& SL & 47 & 4 & $2.96 \pm 0.10$ \\
${ }^{244} \mathrm{Cm}(23.0 \pm 3.7)$ & SI & $52.1 \pm 0.2$ & $1.1 \pm 0.2$ & $11.6 \pm 4.1$ \\
& SII & $55.0 \pm 0.4$ & $2.1 \pm 0.7$ & $37 \pm 12$ \\
& SA & $59.2 \pm 0.7$ & $1.5 \pm 0.5$ & $3.5 \pm 2.4$ \\
& SL & 48 & $5.9 \pm 0.2$ & $48.4 \pm 1.9$ \\
& SI & $54.0 \pm 0.4$ & $2.3 \pm 0.2$ & $7.37 \pm 0.66$ \\
& SII & $58.0 \pm 0.8$ & $2.6 \pm 0.3$ & $5.35 \pm 0.65$ \\
& SL & 49 & $6.82 \pm 0.01$ & $87.95 \pm 0.15$ \\
\hline${ }^{250} \mathrm{Cf}(46.0 \pm 0.2)$ & & & & \\
& & &
\end{tabular}

yield, as expected. Conversely, SI and SII yields decrease with $E_{x}$. The asymmetric modes, in addition, present an additional dependence on the fissility. The SI yield shows a continuous and rather uniform decreasing trend with the fissility, more than with $E_{x}$. This suggests that the SI mode is strongly governed by the height of its fission barrier, correlated with the fissility parameter, more than by the initial $E_{x}$. The SII yield competes with the SI at low $E_{x}$ being always higher than the SI; together, they compensate, as expected, the increasing yield of the SL at higher $E_{x}$. The SA yield, not displayed in the plot, remains very low in ${ }^{240} \mathrm{Pu}$ and ${ }^{244} \mathrm{Cm}$, and negligible in the other systems.

\section{B. Proton even-odd staggering}

The elemental yields of the even- $Z$ fissioning systems show significantly larger production of even- $Z$ fragments than odd$Z$ fragments [11,20-24,40]. This well-known even-odd staggering reflects the probability of breaking proton pairs during the reorganization of the intrinsic structure of the fissioning system [41] and the associated dissipation. The even-odd staggering in even- $Z$ fissioning systems is considered a good indicator of the intrinsic energy of the system at scission as the sum of the excitation energy above the barrier and the energy dissipated along the path from the saddle to the scission point.

In odd- $Z$ fissioning systems, the amplitude of even-odd effect at large asymmetry was reported to be similar to that in even- $Z$ systems for light fragments [42]. For heavy fragments, the even-odd staggering has been reported of similar amplitude, though with an opposite sign (i.e., higher production of odd- $Z$ fragments) [11]. The origin of this staggering in odd- $Z$ systems has been related to the subsequent proton
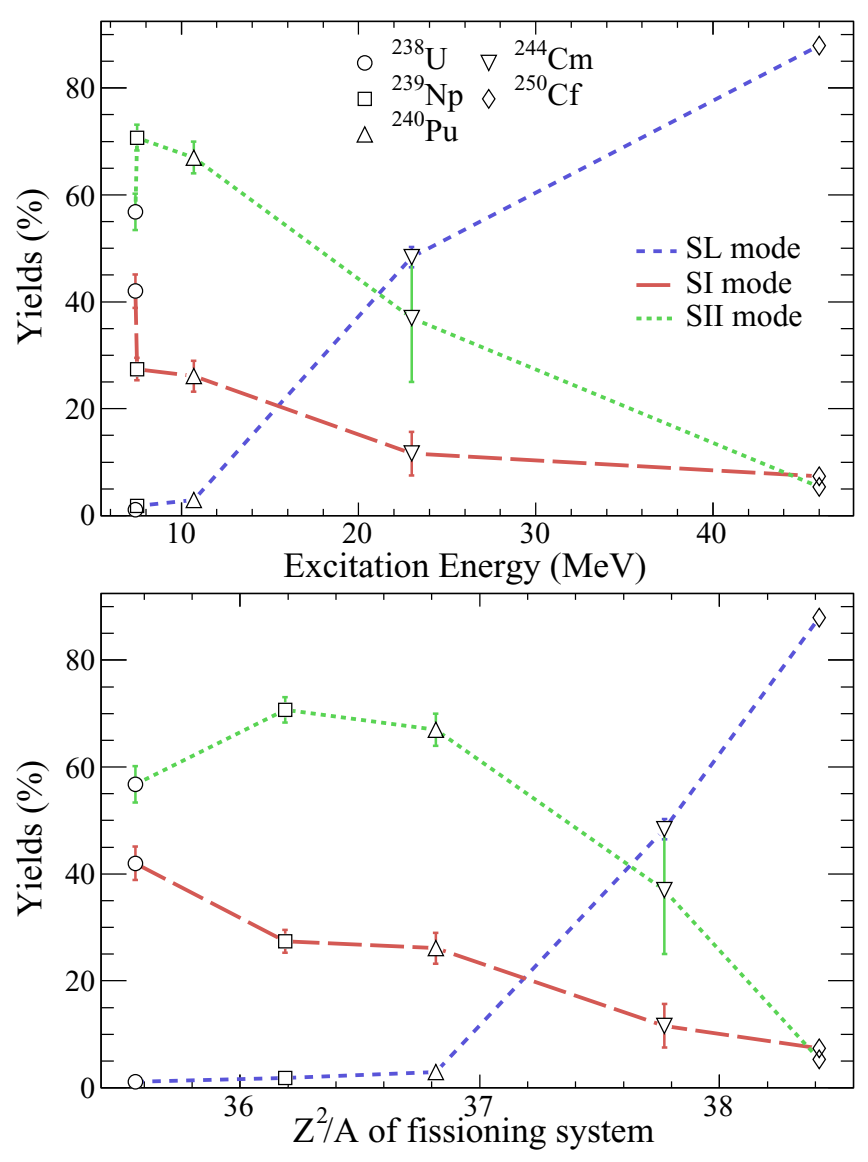

FIG. 5. Yields of the fission modes as a function of the excitation energy of the fissioning system (top), and as a function of the fissility parameter of the fissioning system (bottom).

rearrangement due to the difference in the level densities of the nascent fragments.

In order to disentangle the effect of dissipation (pairbreaking) and unpaired particle rearrangement (following statistical rules), the even-odd effect is studied locally. The local even-odd effect, calculated following Ref. [43], is displayed in Fig. 6 as a function of the fragment nuclear-charge ratio $\left(Z_{1} / Z_{2}\right)$, where $Z_{1}$ and $Z_{2}$ are complementary fission fragments. The nuclear-charge ratio is strongly correlated with the level-density ratio of both fragments.

A strong dependence on the asymmetry is observed, as already discussed in Refs. [11,12]. Even- $Z$ and odd- $Z$ systems show an increasing even-odd staggering with asymmetry, but in the case of the odd- $Z$ system, the sign of the even-odd staggering is reversed, showing higher odd- $Z$ fragment production at large asymmetry.

Both behaviors were described with one single statistical model [11] based on the level densities available in both fission fragments. Nevertheless, ${ }^{238} \mathrm{U},{ }^{239} \mathrm{~Np}$, and ${ }^{240} \mathrm{Pu}$ present an enhancement, not predicted by the previous model, at the $Z$ ratios $\approx 1.16, \approx 1.14$, and $\approx 1.11$, respectively. These maxima correspond to $Z \approx 50$, and suggest structural effects in the potential energy surface that, together with a low intrinsic excitation energy, enhance the production of this element with respect to the smooth trend of the neighboring elements. In 


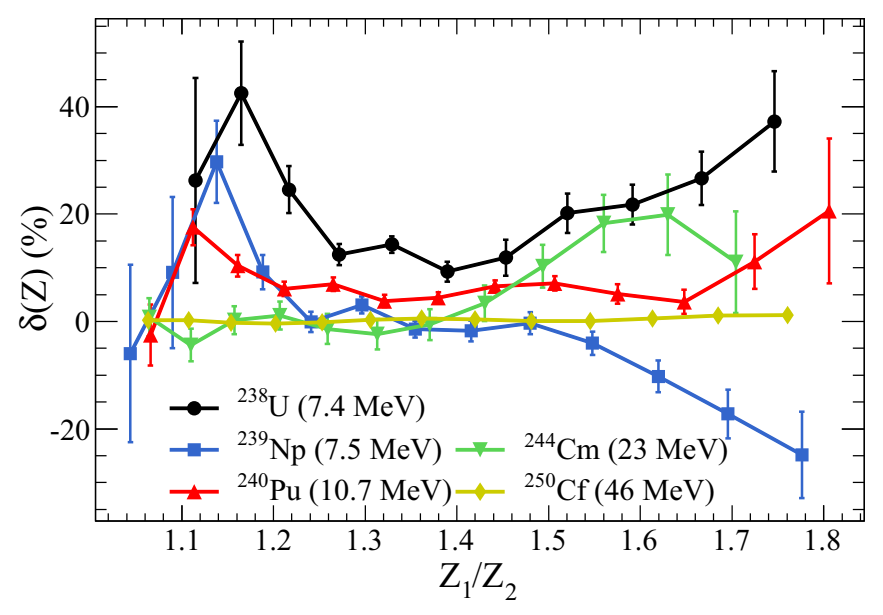

FIG. 6. Local even-odd staggering as a function of the nuclearcharge ratio. Each color represents one fissioning system with its average excitation energy indicated in parentheses.

${ }^{250} \mathrm{Cf}$, the high $E_{x}$ permits the breaking of many proton pairs and the subsequent proton rearrangement proceeds with a reduced influence of the level density, hence, the even-odd effect is observed to be almost zero and constant with respect to the $Z$ ratio.

In order to investigate the interplay between the dynamics of the fission process and the statistical rules of the rearrangement of nucleons, the local even-odd staggering, in absolute values, is studied as a function of the fissility parameter at three different asymmetries: at low asymmetry corresponding to $Z \approx 50$, at moderate asymmetry $\left(Z_{1} / Z_{2}=1.4\right)$, and at large asymmetry $\left(Z_{1} / Z_{2}=1.7\right)$. Figure 7 presents these values compared with previous measurement of $n_{t h}$-induced fission from Refs. [20-24,40,42]. In general, present data show a lower even-odd effect than that in the $n_{t h}$-induced fission, due to the higher $E_{x}$. A decreasing trend is observed with increasing fissility that, in this work, is also associated to increasing $E_{x}$, consistent with higher energy available at the saddle point.

At $Z=50$, both sets of data present similar decreasing trends with increasing fissility, with a shift produced by the difference of $E_{x}$ between them. The odd- $Z$ system ${ }^{239} \mathrm{~Np}$ follows, in this case, the trend of even- $Z$ systems, and with positive sign. This indicates that the structural effect in the potential energy are more important than the level density effect.

At $Z_{1} / Z_{2}=1.4$, the set of data of $n_{t h}$-induced fission exhibits a pronounced decreasing trend. This trend cannot be explained by the influence of the fission barrier height alone, which for ${ }^{250} \mathrm{Cf}$ is only $0.9 \mathrm{MeV}$ lower than for ${ }^{230} \mathrm{Th}$ [44]. Instead, an increasing dissipation with the fissility could explain the drop in $|\delta|[45,46]$. The additional $E_{x}$ of the present data adds to the energy at saddle and permits the breaking of more proton-pairs, decreasing $|\delta|$. Present data differ from the previous data mainly at low fissility. The fact that both sets of data present almost negligible $\delta$ difference in ${ }^{250} \mathrm{Cf}$, despite the large $E_{x}$ difference $\left(\Delta E_{x} \approx 39 \mathrm{MeV}\right.$ ), is consistent with the effect of strong dissipation in ${ }^{250} \mathrm{Cf}$. However, at

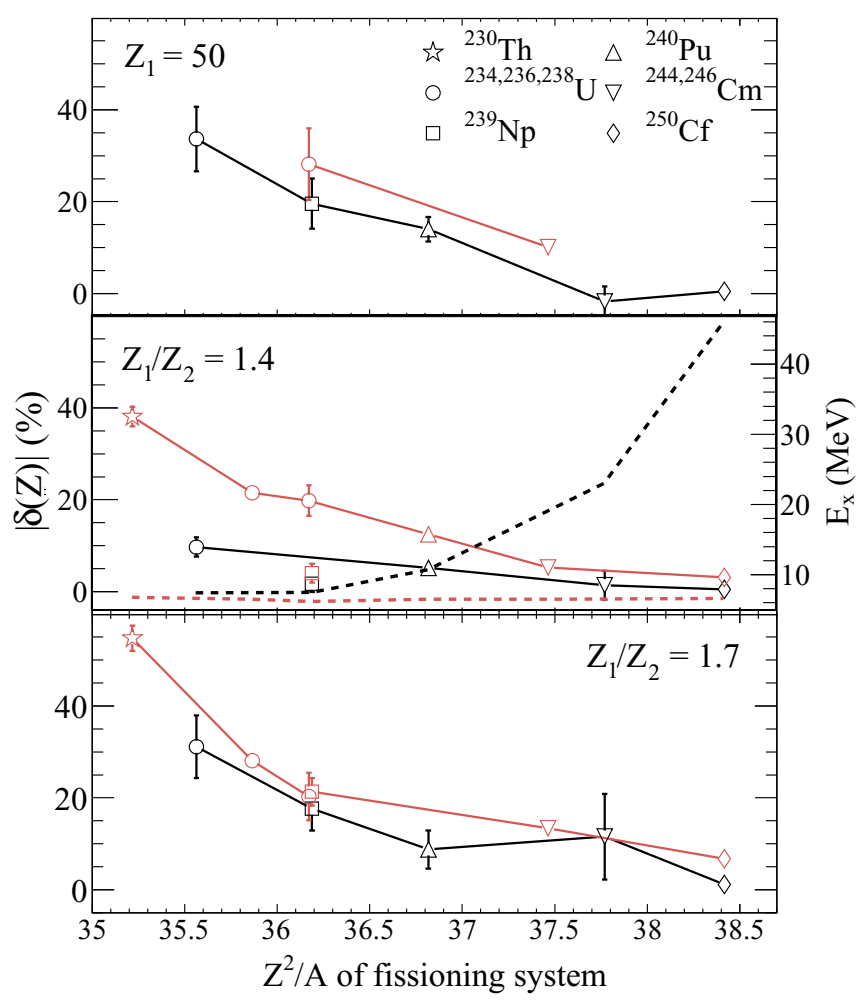

FIG. 7. Local even-odd staggering, in absolute values, as a function of the fissility parameter at different asymmetries. Present data (in black) are compared with previous measurements of thermalneutron induced fission (in red) from Refs. [20-24,40,42]. The excitation energy is shown with dashed lines in the central panel.

low fissility, the large difference in $|\delta|$ between both sets of data are hardly explained by the $E_{x}$ difference of $\approx 1 \mathrm{MeV}$. Concerning ${ }^{239} \mathrm{~Np}$, the even-odd effect is less sensitive to the proton-pair breaking because the system has always one unpaired proton.

At higher $Z$ ratio $\left(Z_{1} / Z_{2}=1.7\right)$, the even-odd effect shows systematically higher values with respect to the moderate asymmetry. This is understood as the influence of the difference in level density between both nascent fragments. In the case of ${ }^{239} \mathrm{~Np}$, with an additional unpaired proton, the amplitude of the staggering is similar to the staggering observed in the fission of even- $Z$ fissioning systems. This shows that, for large asymmetry, the influence of the level density in the fragments dominates the even-odd staggering.

\section{ISOTONIC FISSION-FRAGMENT YIELDS}

The simultaneous measurement of the atomic and mass numbers of the full distribution of fission fragments gives the unique opportunity of extracting the distribution in terms of neutron content, obtained as the sum of the yields of the different isotopes with the same number of neutrons.

Figure 8 presents the isotonic distributions of the five systems investigated in this work. These distributions, contrary to the $Z$ distributions, combine the effect of the neutron sharing at scission with the effect of the excitation energy of the fragments released through neutron evaporation. The 


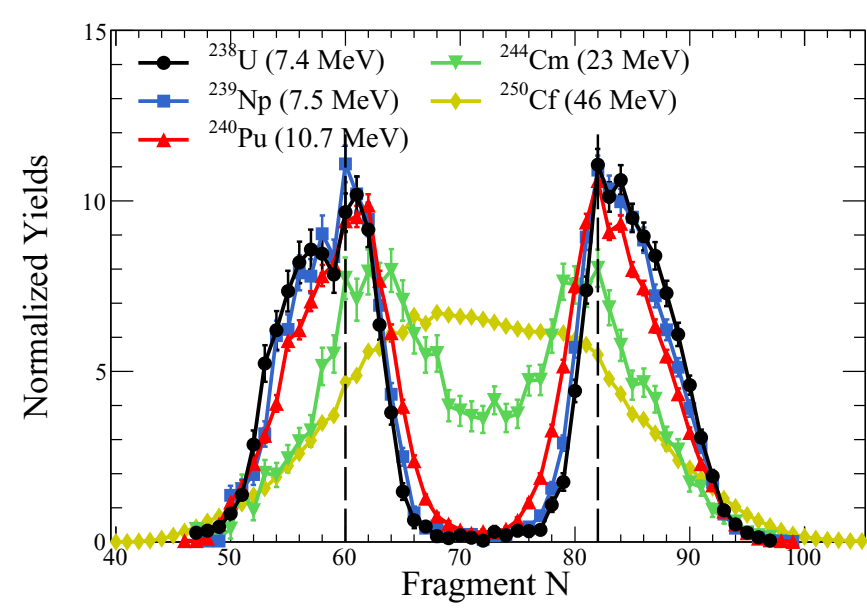

FIG. 8. Fission-fragment neutron distribution. Each color represents one fissioning system with an average excitation energy indicated in parentheses. Dashed lines indicate $N=60$ and $N=82$ (see text for details).

impact of this neutron evaporation is clearly reflected in the distribution of ${ }^{250} \mathrm{Cf}$. The $N$ distribution presents a plateau with systematically higher neutron yields at lighter fragments while the plateau in the $Z$ distribution is flat (Fig. 4). This difference is produced by the neutron evaporation distribution that, at such high $E_{x}$, presents a smooth increasing trend for heavier fragments [38].

The systems at lower $E_{x}-{ }^{238} \mathrm{U},{ }^{239} \mathrm{~Np}$, and ${ }^{240} \mathrm{Pu}-$ present a maximum at $N=82$. In this region, the neutron evaporation reaches a minimum $[14,15,38]$ that determines an endpoint of the neutron-evaporation of heavier fragments and, hence, it produces a maximum in the post neutron-evaporation isotonic distribution. This enhancement is still present in ${ }^{244} \mathrm{Cm}$, although its large $E_{x}$ reduces the impact of this $N=82$ closed shell.

In the light fragments, a clear structure is observed with a maximum yield at $N \approx 60$ for ${ }^{238} \mathrm{U},{ }^{239} \mathrm{~Np}$, and ${ }^{240} \mathrm{Pu}$. In these systems, these isotones are mainly fed by $Z=40$, according to the isotopic distributions, where no shell gaps are expected in $Z \approx 40$ and $N \approx 60[2,47]$. A possible interpretation of the origin of this structure may be the large deformation of the ground state [39] in this nuclei area, reducing the deformation energy released through neutron evaporation from scission to ground-state deformation.

These three systems, ${ }^{238} \mathrm{U},{ }^{239} \mathrm{~Np}$, and ${ }^{240} \mathrm{Pu}$, all of them with 146 neutrons, show clearly different shapes: the heavier the system, the narrower the distribution in both light $[N \in(52,60)]$ and heavy $[N \in(82,90)]$ fragments. These differences reflect the interplay between both the proton and neutron content in the fission process that provides different fissilities.

Figure 9 shows the isotonic yields of ${ }^{238} \mathrm{U},{ }^{239} \mathrm{~Np}$, and ${ }^{240} \mathrm{Pu}$, compared with previous measurements at different excitation energies. ${ }^{238} \mathrm{U}$ is compared with data from Coulombinduced fission [29] at almost the double amount of $E_{x}$. Both sets of data are in good agreement in the light region $(N<60)$, but they clearly differ in the complementary heavy

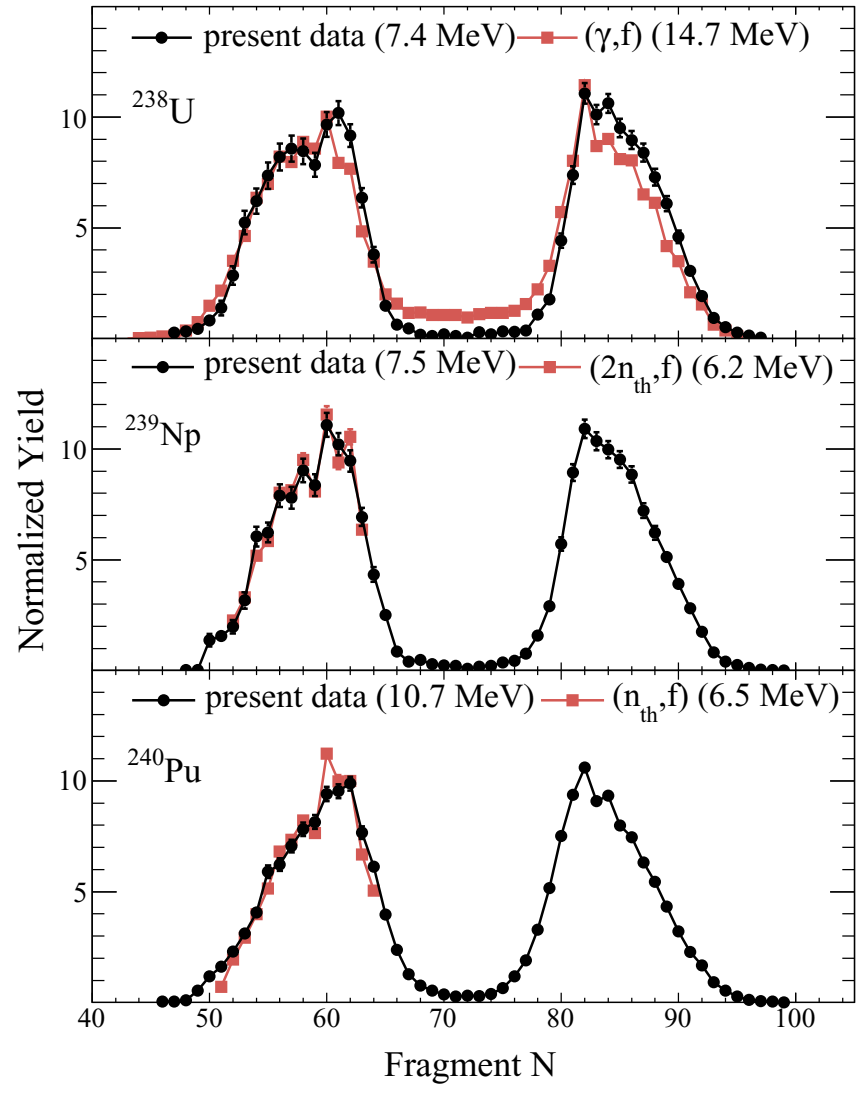

FIG. 9. Present isotonic fission yields of ${ }^{238} \mathrm{U},{ }^{239} \mathrm{~Np}$, and ${ }^{240} \mathrm{Pu}$ (circles) compared with previous measurements (squares) [23,29,48] at different $E_{x}$, indicated within parentheses.

region $(N>82)$. This behavior confirms that the heavy fragment is more likely to receive the additional $E_{x}$, released in neutron evaporation, shifting the distribution in the heavy region more than in the light counterpart, as discussed in Sec. II and in Ref. [18]. The higher $E_{x}$ also smoothes the sharp transition at $N=60$ to lighter fragments and it favors also symmetric fission, feeding the valley of the distribution.

Fission of ${ }^{239} \mathrm{~Np}$ and ${ }^{240} \mathrm{Pu}$ is compared with data from $2 n_{t h}$-induced fission [48] and $n_{t h}$-induced fission [23], respectively. In both cases, previous measurements were limited to light fragments. In ${ }^{239} \mathrm{~Np}$, both sets of data have similar $E_{x}$ as reflected in an overall good agreement. In ${ }^{240} \mathrm{Pu}$, the effect of the higher $E_{x}$ of the present data appears at $N=60$, where the sharp transition to lighter fragments smoothes.

\section{FISSION-FRAGMENT NEUTRON EXCESS}

The isotopic identification permits to investigate fission not only in terms of the neutron and proton content of the fission fragments separately, but also the correlation between them. In this way, the neutron excess of fragments, defined as the average number of neutrons per proton, is a suitable observable.

A structureless scission-point model (LD-SP) predicts a sharing of nucleons at scission dominated by the interplay between the Coulomb repulsion and nuclear interactions. This 


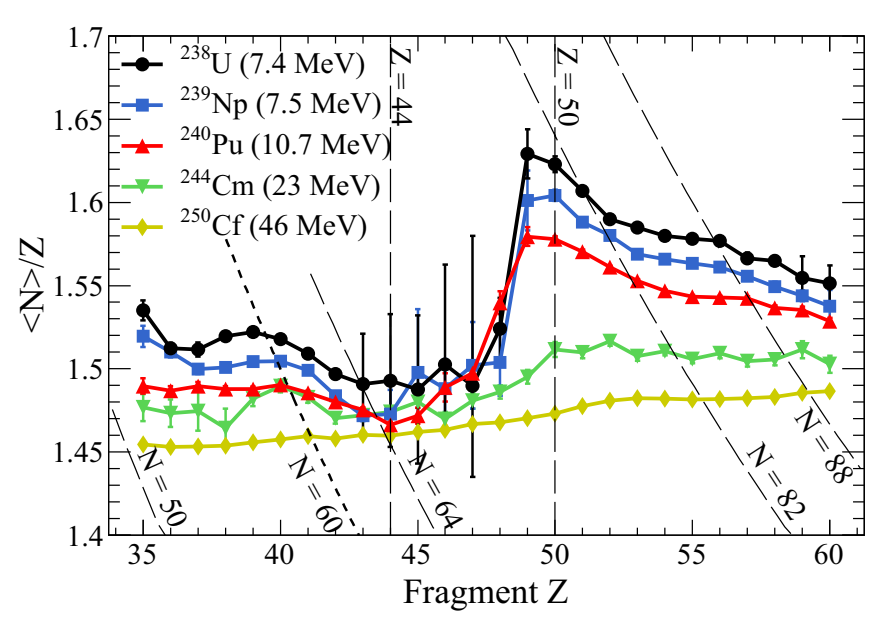

FIG. 10. Fission-fragment neutron excess as a function of the fission-fragment nuclear charge. Spherical shells $Z=50, N=$ 50,82 , and deformed shells $Z=44, N=64,88$ are indicated with dashed lines. The isotonic line $N=60$ is also presented.

results in fragments with a smoothly increasing number of neutrons as a function of $Z$ [38].

However, this trend does not agree with previous measurements at low excitation energy [27,29]. In this energy regime, the heavy fragment has a strong and a saw-tooth-like excess of neutrons with respect to the LD-SP model prediction, while the light fragment is less neutron rich. Furthermore, some particular $(Z, N)$ configurations of the nascent fragments are favored, such as $(50,82)$ (see Fig. 2). The neutron evaporation, which also affects the measured neutron excess, reflects the sharing of excitation energy at the scission-point and it is also influenced by shell effects, as shown in the previous section. The neutron excess reflects all these effects and is, therefore, an observable sensitive to structure effects in the sharing of protons and neutrons between the fragments independent of their production yields.

Figure 10 shows the average neutron excess of the five fissioning systems investigated in this work. The vertical lines indicate the proton number associated with the deformed and spherical shells $Z=44,50$, respectively. The curved diagonals indicate the position of the neutron excess centered on spherical and deformed shell neutron numbers $N=50,82$, and $N=64,88$, respectively, as expected from calculations based on the model by Strutinsky [2,47]. The neutron excess for neutron number $N=60$ is also shown.

The neutron excess of ${ }^{250} \mathrm{Cf}$ exhibits a smoothly increasing behavior with no strong structure effects. This is inline with the expectation of the liquid-drop scission-point model, indicating the hindrance of shell effects with excitation energy. In a fissioning system produced at $46 \mathrm{MeV}$ of excitation energy, it is possible that neutron evaporation would arise before scission, leading to fission with a reduced excitation energy [36], and a consequent shell structure appearing in the fission-fragment properties. The smooth behavior of the neutron-excess without any structural effect as observed in lower energy fission, indicates that the multichance fission is hindered. This is consistent with de-excitation model calculations, such as General Description of Fission Observables (GEF) [49].

Charge polarization is observed in ${ }^{244} \mathrm{Cm}$ with two different trends: a stabilization in the heavy region with a maximum value at $Z \approx 50$, close to a spherical shell, and a lower $N / Z$ in the light region, where a local maximum is observed around $Z \approx 40$, also present in the cooler systems.

The systems measured at lower $E_{x}-{ }^{240} \mathrm{Pu},{ }^{239} \mathrm{~Np}$, and ${ }^{238} \mathrm{U}$ - present a strong charge polarization with a steep increase shape at $Z=49$. The maximum value around $Z \approx 50$ breaks the overall increasing trend observed in the systems with higher $E_{x}$. Such a large neutron excess may be understood as the influence of the doubly magic nucleus ${ }^{132} \mathrm{Sn}$, which corresponds to neutron excess of 1.64. The low neutron excess of the complementary $Z$ fragment compensates the excess of neutrons of the heavy fragment, even after postscission neutron evaporation. As the excitation energy increases, the steep increase around $Z \approx 50$ is less pronounced, in contrary to the neutron excess of the light fragments which remain very similar.

A general shift is observed between different systems. This shift is inline with the $N / Z$ of the fissioning system. This is clearly observed for ${ }^{238} \mathrm{U}$ and ${ }^{239} \mathrm{~Np}$, where both sets of data show very similar behavior but shifted by $\approx 0.02$, that corresponds to the $N / Z$ difference of the initial fissioning systems.

The $N / Z$ ratio reveals that the $N=82$ maximum observed in Fig. 8 is correlated with $Z \approx 51,52,53$ in ${ }^{238} \mathrm{U},{ }^{239} \mathrm{~Np}$, and

${ }^{240} \mathrm{Pu}$, respectively. These values are shifted from the maxima observed in the $Z$ distributions, $Z=52,53,54$ (Fig. 4), which confirms that this maximum at $N=82$ is not related with the partition at scission but with the endpoint of the neutron evaporation, as indicated in the previous section.

Concerning the light-fragment region, all the systems converge to a general minimum at $(Z, N) \approx(44,64)$ that corresponds to deformed closed shells. The local maximum observed at $Z \approx 40$ is consistent with lower neutron evaporation in a region where the ground state becomes very deformed, which coincides with the maximum at $N \approx 60$ in Fig. 8 .

A few general aspects can be highlighted: (i) There is a clear trend towards $(Z, N)=(50,82)$ that $E_{x}$ reduces along the $Z=50$ line. (ii) There is a general minimum at $(Z, N)=$ $(44,64)$ that coincides with deformed closed shells. (iii) There is a local maximum around $N \approx 60$ related to low neutron evaporation probabilities.

\section{TOTAL NEUTRON MULTIPLICITY}

The total neutron multiplicity, averaged over the nuclear charge of complementary fragments $\left(\left\langle v_{\text {tot }}\right\rangle\right)$, is obtained as the difference between the number of neutrons of the compound system $\left(N_{\mathrm{CN}}\right)$ and the sum of the average neutron content of two complementary $Z$ fragments:

$$
\left\langle v_{\text {tot }}\right\rangle\left(Z_{1}\right)=N_{\mathrm{CN}}-\left(\langle N\rangle\left(Z_{1}\right)+\langle N\rangle\left(Z_{\mathrm{CN}}-Z_{1}\right)\right) .
$$

Figure 11 presents the average total neutron multiplicity as a function of the fragment $Z$ for the five fissioning systems. The systems at lower $E_{x}-{ }^{238} \mathrm{U},{ }^{239} \mathrm{~Np}$, and ${ }^{240} \mathrm{Pu}$ - show a 


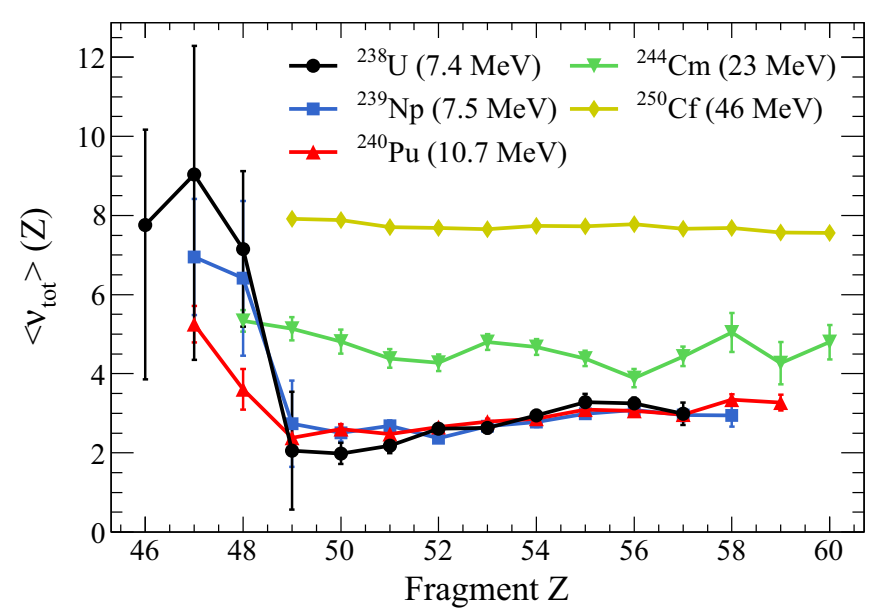

FIG. 11. Average total neutron multiplicity as a function of the fission-fragment nuclear charge.

large neutron multiplicity at symmetry that suggests large deformations at scission, consistent with the description of the superlong symmetric fission mode [3]. These systems also show a minimum at $Z \approx 50$, suggesting small deformations associated with spherical closed shells, $Z=50$ and $N=82$. Then, the neutron multiplicity shows a smooth increasing trend up to $Z \approx 55$, where it reaches a maximum that may be related to the influence of a deformed shell $N \approx 88$ [2].

The systems with higher $E_{x},{ }^{244} \mathrm{Cm}$ and ${ }^{250} \mathrm{Cf}$, have larger average neutron multiplicities without increase at symmetry. In ${ }^{250} \mathrm{Cf}$, a difference of $\approx 20 \mathrm{MeV}$ between the total excitation energy (TXE) of symmetric and asymmetric splits was observed [38], however, this difference is not visible in the neutron multiplicity, which shows a constant trend as a function of $Z$. In addition, as discussed in the previous section, the pre-scission neutron multiplicity is estimated to be relatively small $\left(\langle v\rangle^{\text {pre }}=1.15\right)[5]$. This indicates that, at such high $E_{x}=46 \mathrm{MeV}$ and angular momentum $L=15 \hbar$ (following the Bass description [50]), this asymmetry dependence of TXE

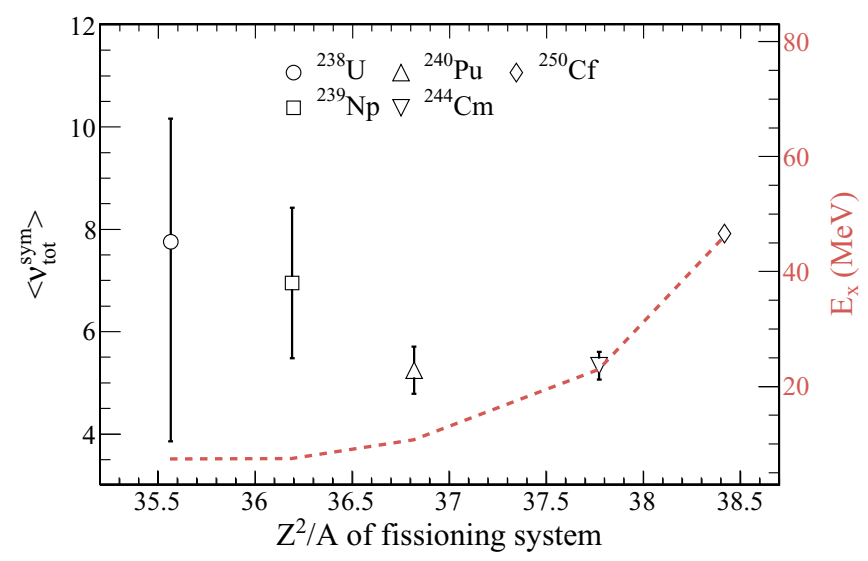

FIG. 12. Average total neutron multiplicity at symmetry as a function of the fissility parameter of the fissioning system. The excitation energy of the fissioning systems is indicated with a dashed line.
TABLE II. Average total neutron multiplicity. The average excitation energies are also shown.

\begin{tabular}{ccc}
\hline \hline System & $\left\langle E_{x}\right\rangle(\mathrm{MeV})$ & $\left\langle v_{\text {tot }}\right\rangle$ \\
\hline${ }^{238} \mathrm{U}$ & 7.4 & $2.78 \pm 0.06$ \\
${ }^{239} \mathrm{~Np}$ & 7.5 & $2.73 \pm 0.04$ \\
${ }^{240} \mathrm{Pu}$ & 10.7 & $2.87 \pm 0.03$ \\
${ }^{244} \mathrm{Cm}$ & 23.0 & $4.59 \pm 0.07$ \\
${ }^{250} \mathrm{Cf}$ & 46.0 & $7.72 \pm 0.02$ \\
\hline \hline
\end{tabular}

must be reflected in the $\gamma$ de-excitation and/or in the kinetic energy of the evaporated neutrons.

Figure 12 shows the total neutron multiplicity at symmetry as a function of the fissility of the fissioning system. The systems at low $E_{x}(\mathrm{U}, \mathrm{Np}$, and $\mathrm{Pu})$ show a decreasing trend with the fissility, whereas the excitation energy in not increasing significantly, which indicates that the excitation energy of fragments at symmetry reduces with the fissility. In $\mathrm{Cm}$ and $\mathrm{Cf}$, the high increasing $E_{x}$ compensates this trend and the multiplicity increases.

The mean value of the total neutron evaporation, along with the full range of the fragment distributions, is presented for each fissioning system in Table II. The increase of $E_{x}$ is reflected in a higher neutron multiplicity with a rather linear behavior for ${ }^{240} \mathrm{Pu},{ }^{244} \mathrm{Cm}$, and ${ }^{250} \mathrm{Cf}$. This confirms that, on average, the neutron multiplicity is a good indicator of the excitation energy of the fissioning system.

\section{CONCLUSIONS}

The high quality of fission data in the inverse kinematics provides new observables that give access to important information on the fission process. In this work, fission is investigated in terms of fragment-related observables of five fissioning systems- ${ }^{238} \mathrm{U},{ }^{239} \mathrm{~Np},{ }^{240} \mathrm{Pu},{ }^{244} \mathrm{Cm}$, and ${ }^{250} \mathrm{Cf}-$ at different excitation energies between 7.4 and $46 \mathrm{MeV}$. In addition to the isotopic fission yields [37], fission modes and proton even-odd staggering are investigated, full isotonic distributions, neutron excess, and total neutron multiplicities are obtained, some of them for first time.

The $Z=50$ yield reveals an unexpected stability against small variations of $E_{x}$ at scission, reflected in the complementary $Z=42$ yield for ${ }^{238} \mathrm{U}$. Related to this, the Standard $I$ mode amplitude shows a clear trend with the fissility parameter, linked to the height of the fission barrier, while the symmetric Super Long mode proves to be very sensitive to the initial excitation energy of the system.

The proton even-odd staggering exhibits a strong dependence on the fragment asymmetry: the stronger the difference of the level densities between both fragments, the higher the even-odd effect. For moderate $Z$ asymmetry, the evenodd effect appears to be more sensitive to the fissility than to the initial $E_{x}$. The comparison with $n_{t h}$-induced fission confirms that the dissipation from the saddle to the scission point dominates the even-odd staggering in systems with high fissility.

The isotonic distributions at low excitation energy shows a maximum at $N=82$, presumably driven by a minimum 
of neutron evaporation due to the spherical closed shell. An additional maximum is observed at $N \approx 60$. The comparison with higher-energy data proves that the excess of the excitation energy is driven by the heavy fragment, released through neutron evaporation.

The impact of the spherical closed shells at $Z=50$ and $N=82$ appears in the neutron excess, with a high neutron content in the region of the doubly-magic nucleus ${ }^{132} \mathrm{Sn}$. In ${ }^{250} \mathrm{Cf}$, the high $E_{x}$ and the neutron evaporation prevents the survival of any major structure effects. Nevertheless, the convergence of the systems to similar $N / Z$ values in $Z=44$ suggests also the effect of deformed closed shells. The neutron excess around $Z \approx 40$ reflects lower neutron evaporation that coincides with the maximum yield in $N \approx 60$.

The total neutron multiplicity suggests systematically larger deformations at symmetry, and it reveals the impact of spherical shells at $Z=50$ with a minimum in evaporation. At sufficiently high excitation energies, the additional $E_{x}$ is fully released through neutron evaporation, reflected in the linearity between the averaged total neutron evaporation and $E_{x}$.

This work is part of a recent initiative developed at different places, aiming at improving experimental description of fission. The experimental conditions are based on inverse kinematics and surrogate reactions, combined with magnetic spectrometers [27,29], and are inspired by the work initiated at GSI $[8,19]$. These new kinds of campaigns provide highprecision and complete yield data, together with an unprecedented ensemble of observables gathered in one single experiment. This will initiate strong improvements in the theoretical description of the fission process [49,51].

\section{ACKNOWLEDGMENTS}

The authors thank Dr. A. Navin for his contribution in the experiment. This work was partially supported by the Spanish Ministry of Research and Innovation under the budget items FPA2010-22174-C02-01 and RYC-2012-11585.
[1] N. Bohr and J. A. Wheeler, Phys. Rev. 56, 426 (1939).

[2] B. D. Wilkins et al., Phys. Rev. C 14, 1832 (1976).

[3] U. Brosa, S. Grossman, and A. Müller, Phys. Rep. 197, 167 (1990).

[4] P. Möller, J. Randrup, and A. J. Sierk, Phys. Rev. C 85, 024306 (2012).

[5] K.-H. Schmidt et al., Nucl. Data Sheets 131, 107 (2016).

[6] D. Regnier, N. Dubray, N. Schunck, and M. Verriere, Phys. Rev. C 93, 054611 (2016).

[7] K. F. Flynn et al., Phys. Rev. C 5, 1725 (1972).

[8] C. Böckstiegel et al., Nucl. Phys. A 802, 12 (2008).

[9] B. Jurado, K.-H. Schmidt, and J. Benlliure, Phys. Lett. B 553, 186 (2003).

[10] J. L. Rodríguez-Sánchez et al., Phys. Rev. C 94, 061601(R) (2016).

[11] S. Steinhäuser et al., Nucl. Phys. A 634, 89 (1998).

[12] M. Caamaño et al., J. Phys. G: Nucl. Part. Phys. 38, 035101 (2011).

[13] F. Pleasonton, R. L. Ferguson, and H. Schmitt, Phys. Rev. C 6, 1023 (1972).

[14] R. Müller, A. A. Naqvi, F. Kappeler, and F. Dickmann, Phys. Rev. C 29, 885 (1984).

[15] A. A. Naqvi, F. Kappeler, F. Dickmann, and R. Muller, Phys. Rev. C 34, 218 (1986).

[16] R. Capote et al., Nucl. Data Sheets 131, 1 (2016).

[17] O. Litaize and O. Serot, Phys. Rev. C 82, 054616 (2010).

[18] K.-H. Schmidt and B. Jurado, Phys. Rev. Lett. 104, 212501 (2010).

[19] K.-H. Schmidt et al., Nucl. Phys. A 665, 221 (2000).

[20] W. Lang et al., Nucl. Phys. A 345, 34 (1980).

[21] U. Quade et al., Nucl. Phys. A 487, 1 (1988).

[22] M. Djebara et al., Nucl. Phys. A 496, 346 (1989).

[23] C. Schmitt et al., Nucl. Phys. A 430, 21 (1984).

[24] J. P. Bocquet, R. Brissot, H. R. Faust, M. Fowler, J. Wilhelmy, M. Asghar, and M. Djebara, Z. Phys. A 335, 41 (1990).

[25] H. Naik et al., Nucl. Phys. A 612, 143 (1997).

[26] A. Bail, O. Serot, L. Mathieu, O. Litaize, T. Materna, U. Koster, H. Faust, A. Letourneau, and S. Panebianco, Phys. Rev. C 84, 034605 (2011).
[27] M. Caamaño, O. Delaune, F. Farget, X. Derkx, K. H. Schmidt, L. Audouin, C. O. Bacri, G. Barreau, J. Benlliure, E. Casarejos, A. Chbihi, B. Fernandez-Dominguez, L. Gaudefroy, C. Golabek, B. Jurado, A. Lemasson, A. Navin, M. Rejmund, T. Roger, A. Shrivastava, and C. Schmitt, Phys. Rev. C 88, 024605 (2013).

[28] J. L. Rodríguez-Sánchez et al., Phys. Rev. C 91, 064616 (2015).

[29] E. Pellereau, J. Taieb, A. Chatillon, H. Alvarez-Pol, L. Audouin, Y. Ayyad, G. Belier, J. Benlliure, G. Boutoux, M. Caamano, E. Casarejos, D. Cortina-Gil, A. Ebran, F. Farget, B. FernandezDominguez, T. Gorbinet, L. Grente, A. Heinz, H. Johansson, B. Jurado, A. Kelic-Heil, N. Kurz, B. Laurent, J. F. Martin, C. Nociforo, C. Paradela, S. Pietri, J. L. Rodriguez-Sanchez, K. H. Schmidt, H. Simon, L. Tassan-Got, J. Vargas, B. Voss, and H. Weick, Phys. Rev. C 95, 054603 (2017).

[30] G. Boutoux, B. Jurado et al., Phys. Lett. B 712, 319 (2012).

[31] C. Rodríguez-Tajes et al., Phys. Rev. C 89, 024614 (2014).

[32] J. Khuyagbaatar, D. J. Hinde, I. P. Carter, M. Dasgupta, C. E. Dullmann, M. Evers, D. H. Luong, R. duRietz, A. Wakhle, E. Williams, and A. Yakushev, Phys. Rev. C 91, 054608 (2015).

[33] K. Nishio et al., Phys. Lett. B 748, 89 (2015).

[34] R. Léguillon, K. Nishio et al., Phys. Lett. B 761, 125 (2016).

[35] Q. Ducasse, B. Jurado et al., Phys. Rev. C 94, 024614 (2016).

[36] K. Hirose, K. Nishio et al., Phys. Rev. Lett. 119, 222501 (2017).

[37] D. Ramos, M. Caamano, F. Farget, C. Rodriguez-Tajes, L. Audouin, J. Benlliure, E. Casarejos, E. Clement, D. Cortina, O. Delaune, X. Derkx, A. Dijon, D. Dore, B. FernandezDominguez, G. deFrance, A. Heinz, B. Jacquot, A. Navin, C. Paradela, M. Rejmund, T. Roger, M. D. Salsac, and C. Schmitt, Phys. Rev. C 97, 054612 (2018).

[38] M. Caamano, F. Farget, O. Delaune, K. H. Schmidt, C. Schmitt, L. Audouin, C. O. Bacri, J. Benlliure, E. Casarejos, X. Derkx, B. Fernandez-Dominguez, L. Gaudefroy, C. Golabek, B. Jurado, A. Lemasson, D. Ramos, C. Rodriguez-Tajes, T. Roger, and A. Shrivastava, Phys. Rev. C 92, 034606 (2015).

[39] M. Caamaño and F. Farget, Phys. Lett. B 770, 72 (2017).

[40] D. Rochman et al., Nucl. Phys. A 710, 3 (2002). 
[41] H. Nifenecker et al., Z. Phys. A 308, 39 (1982).

[42] I. Tsekhanovich et al., Nucl. Phys. A 688, 633 (2001).

[43] B. L. Tracy et al., Phys. Rev. C 5, 222 (1972).

[44] S. Bjørnholm and J. E. Lynn, Rev. Mod. Phys. 52, 725 (1980).

[45] B. Bouzid et al., J. Phys. G 24, 1029 (1998).

[46] F. Rejmund et al., Nucl. Phys. A 678, 215 (2000).

[47] V. M. Strutinsky, Nucl. Phys. A 95, 420 (1967).
[48] G. Martinez et al., Nucl. Phys. A 515, 433 (1990).

[49] C. Schmitt, K.-H. Schmidt, and B. Jurado, Phys. Rev. C 98, 044605 (2018).

[50] R. Bass, Nuclear Reactions with Heavy Ions (Springer-Verlag, Berlin, 1980).

[51] K.-H. Schmidt and B. Jurado, Rep. Prog. Phys. 81, 106301 (2018). 